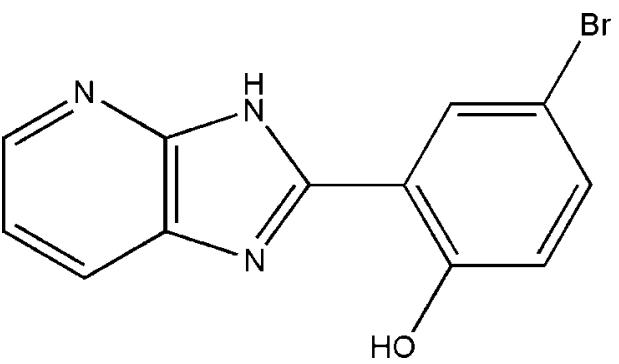

\title{
Crystal structure of 4-bromo-2-(1H- imidazo[4,5-b]pyridin-2-yl)phenol
}

\section{Kamel Ouari}

Laboratoire d'Electrochimie, d'Ingénierie Moléculaire et de Catalyse Redox, Faculty of Technology, University of Ferhat Abbas Sétif-1, 19000 Sétif, Algeria. *Correspondence e-mail: k_ouari@yahoo.fr

Received 6 November 2015; accepted 19 November 2015

In the title compound, $\mathrm{C}_{12} \mathrm{H}_{8} \mathrm{BrN}_{3} \mathrm{O}$, the 4-bromophenol ring is coplanar with the planar imidazo[4,5-b]pyridine moiety (r.m.s deviation $=0.015 \AA$ ), making a dihedral angle of $1.8(2)^{\circ}$. There is an intramolecular $\mathrm{O}-\mathrm{H} \cdots \mathrm{N}$ hydrogen bond forming an $S(6)$ ring motif. In the crystal, molecules are linked via $\mathrm{N}-\mathrm{H} \cdots \mathrm{N}$ and $\mathrm{O}-\mathrm{H} \cdots \mathrm{Br}$ hydrogen bonds, forming undulating sheets parallel to $(10 \overline{2})$. The sheets are linked by $\pi-\pi$ interactions [inter-centroid distance $=3.7680(17) \AA$ ], involving inversion-related molecules, forming a three-dimensional structure.

Keywords: crystal structure; 2,3-diaminopyridine; 5-bromo-2-hydroxy-1salycilaldehyde; hydrogen bonding.

CCDC reference: 1437912

\section{Related literature}

For some recent examples of transition metal complexes of Schiff bases, see: Ouari et al. (2015b); Benghanem et al. (2012); Basu et al. (2010). For the biological activity of Schiff bases, see: Ylldiz et al. (2015); Salama et al. (2015); Zayed et al. (2015). For the photoluminescence of the title compound, see: Köse et al. (2015); Pal et al. (2015); Ray et al. (2014). For the literature method used to prepare the title compound, see: Ouari et al. (2015a). For the crystal structure of a related compound, see: Belguedj et al. (2015).

\subsection{Data collection}

Nonius KappaCCD diffractometer Absorption correction: multi-scan (MULABS in PLATON; Spek, 2009)

$T_{\min }=0.457, T_{\max }=0.721$

\subsection{Refinement}

$R\left[F^{2}>2 \sigma\left(F^{2}\right)\right]=0.043$

$w R\left(F^{2}\right)=0.111$

$S=1.02$

3017 reflections

159 parameters
$V=1038.28(9) \AA^{3}$

$Z=4$

Mo $K \alpha$ radiation

$\mu=3.94 \mathrm{~mm}^{-1}$

$T=193 \mathrm{~K}$

$0.25 \times 0.20 \times 0.05 \mathrm{~mm}$

Table 1

Hydrogen-bond geometry $\left(\AA{ }^{\circ}\right)$.

\begin{tabular}{lllll}
\hline$D-\mathrm{H} \cdots A$ & $D-\mathrm{H}$ & $\mathrm{H} \cdots A$ & $D \cdots A$ & $D-\mathrm{H} \cdots A$ \\
\hline $\mathrm{O} 1-\mathrm{H} 1 \cdots \mathrm{N} 2$ & 0.84 & 1.90 & $2.640(3)$ & 147 \\
$\mathrm{O} 1-\mathrm{H} 1 \cdots \mathrm{Br}^{\mathrm{i}}$ & 0.84 & 2.91 & $3.478(2)$ & 127 \\
$\mathrm{~N} 1-\mathrm{H} 1 N \cdots \mathrm{N} 3^{\mathrm{ii}}$ & $0.92(4)$ & $2.11(4)$ & $3.010(4)$ & $168(3)$ \\
\hline
\end{tabular}

Symmetry codes: (i) $x-1,-y+\frac{3}{2}, z-\frac{1}{2}$; (ii) $-x+2,-y+2,-z+2$.

Data collection: COLLECT (Nonius, 1998); cell refinement: DENZO (Nonius, 1998); data reduction: DENZO; program(s) used to solve structure: SHELXS97 (Sheldrick, 2008); program(s) used to refine structure: SHELXL97 (Sheldrick, 2008); molecular graphics: Mercury (Macrae et al., 2008); software used to prepare material for publication: SHELXL97 and PLATON (Spek, 2009).

\section{Acknowledgements}

The author gratefully acknowledges financial support from the Algerian Ministry of Higher Education and Scientific Research. He also acknowledges the help of Dr Jean Weiss (CLAC) at the University of Strasbourg, France. 
Supporting information for this paper is available from the IUCr electronic archives (Reference: SU5238).

\section{References}

Basu, S., Gupta, G., Das, B. \& Rao, K. M. (2010). J. Organomet. Chem. 695, 2098-2104.

Belguedj, R., Bouacida, S., Merazig, H., Chibani, A. \& Bouraiou, A. (2015). Acta Cryst. E71, o131-o132.

Benghanem, F., Keraghel, S., Chahmana, S., Ourari, A. \& Brelot, L. (2012). Acta Cryst. E68, o2188-o2189.

Salama, H. E., Saad, G. R. \& Sabaa, M. W. (2015). Int. J. Biol. Macromol. 79, 996-1003.

Köse, M., Ceyhan, G., Tümer, M., Demirtaş, İ., Gönül, İ. \& McKee, V. (2015). Spectrochim. Acta Part A, 137, 477-485.
Macrae, C. F., Bruno, I. J., Chisholm, J. A., Edgington, P. R., McCabe, P., Pidcock, E., Rodriguez-Monge, L., Taylor, R., van de Streek, J. \& Wood, P. A. (2008). J. Appl. Cryst. 41, 466-470.

Nonius (1998). COLLECT and DENZO. Nonius BV. Delft, The Netherlands. Ouari, K., Bendia, S., Merzougui, M. \& Bailly, C. (2015a). Acta Cryst. E71, o51-052.

Ouari, K., Bendia, S., Weiss, J. \& Bailly, C. (2015b). Spectrochim. Acta A Mol. Biomol. Spectrosc. 135, 624-631.

Pal, M. K., Kushwah, N., Wadawale, A. P., Manna, D., Sudarsan, V., Ghanty, T. K. \& Jain, V. K. (2015). J. Organomet. Chem. 776, 98-106.

Ray, S., Konar, S., Jana, A., Das, K., Dhara, A., Chatterjee, S. \& Kar, S. K. (2014). J. Mol. Struct. 1058, 213-220.

Sheldrick, G. M. (2008). Acta Cryst. A64, 112-122.

Spek, A. L. (2009). Acta Cryst. D65, 148-155.

Yıldız, M., Karpuz, Ö., Özge, , Zeyrek, C. T., Boyacıŏ̆lu, B., Dal, H., Demir, N., Yıldırım, N. \& Ünver, H. (2015). J. Mol. Struct. 1094, 148-160.

Zayed, E. M. \& Zayed, M. A. (2015). Spectrochim. Acta A Mol. Biomol. Spectrosc. 143, 81-90. 


\section{supporting information}

Acta Cryst. (2015). E71, o991-0992 [https://doi.org/10.1107/S2056989015022197]

\section{Crystal structure of 4-bromo-2-(1H-imidazo[4,5-b]pyridin-2-yl)phenol}

\section{Kamel Ouari}

\section{S1. Comment}

Coordination chemistry of transition metal complexes with Schiff base ligands is an important and fascinating branch of chemistry (Ouari et al., 2015b; Benghanem et al., 2012; Basu et al., 2010). A literature survey revealed that this kind of compound possesses diverse biological activities such as antibiotic (Yıldiz et al., 2015) and antimicrobial (Salama et al., 2015; Zayed et al., 2015). The photoluminescence of the title compound has been reported (Köse et al., 2015; Pal et al., 2015; Ray et al., 2014).

The molecular structure of the title compound is shown in Fig. 1. The bond distances and angles are normal and similar to those in related compounds (Belguedj et al., 2015).

In the crystal, molecules are linked via $\mathrm{N}-\mathrm{H} \cdots \mathrm{N}$ and $\mathrm{O}-\mathrm{H} \cdots \mathrm{Br}$ hydrogen bonds forming undulating sheets parallel to $(10 \overline{2})$; see Table 1 and Fig. 2 . The sheets are linked by $\pi-\pi$ interactions $\left[\mathrm{Cg} 2 \cdots \operatorname{Cg} 3{ }^{\mathrm{i}}=3.7680\right.$ (17) $\AA, \mathrm{Cg} 2$ and $\mathrm{Cg} 3$ are the centroids of rings $\mathrm{N} 3 / \mathrm{C} 8-\mathrm{C} 12$ and $\mathrm{C} 1-\mathrm{C} 6$, symmetry code: (i) $-\mathrm{x}+1,-\mathrm{y}+2,-\mathrm{z}+2$ ], forming a three-dimensional structure.

\section{S2. Synthesis and crystallisation}

The title compound was prepared following a literature method (Ouari et al., 2015a). To a MeOH solution (15 ml) of 5bromosalicylaldehyde $(0.122 \mathrm{~g}, 1 \mathrm{mmol})$ was added drop wise to a $\mathrm{MeOH}$ solution $(5 \mathrm{ml})$ of 2,3-diaminopyridine $(0.109$ $\mathrm{g}, 1 \mathrm{mmol})$. The mixture was refluxed with constant stirring under a nitrogen atmosphere for $3 \mathrm{~h}$, yielding an abundant orange precipitate that was collected by filtration. The product was washed with methanol $(3 \times 5 \mathrm{ml})$ then with diethyl ether $(3 \times 5 \mathrm{ml})$ and dried under vacuum for $4 \mathrm{~h}$. Orange crystals of the title compound, suitable for X-ray diffraction analysis, were obtained after two weeks by slow evaporation of the DMSO solvent (yield: 70\%; m.p.: 528-531 K).

\section{S3. Refinement}

Crystal data, data collection and structure refinement details are summarized in Table 2. The iminium $\mathrm{H}$ atom was located from a difference Fourier map and freely refined. The $\mathrm{OH}$ and $\mathrm{C}$-bound $\mathrm{H}$ atoms were included in calculated positions and treated as riding atoms: $\mathrm{O}-\mathrm{H}=0.82 \AA, \mathrm{C}-\mathrm{H}=0.95-0.99 \AA$ with $\mathrm{U}_{\mathrm{iso}}(\mathrm{H})=1.5 \mathrm{U}_{\mathrm{eq}}(\mathrm{O})$ and $1.2 \mathrm{U}_{\mathrm{eq}}(\mathrm{C})$. 




Figure 1

The molecular structure of the title compound, with atom labelling scheme. Displacement ellipsoids are drawn at the 50\% probability level. The intramolecular $\mathrm{O}-\mathrm{H} \cdots \mathrm{N}$ hydrogen bond is shown as a dashed line (see Table 1).

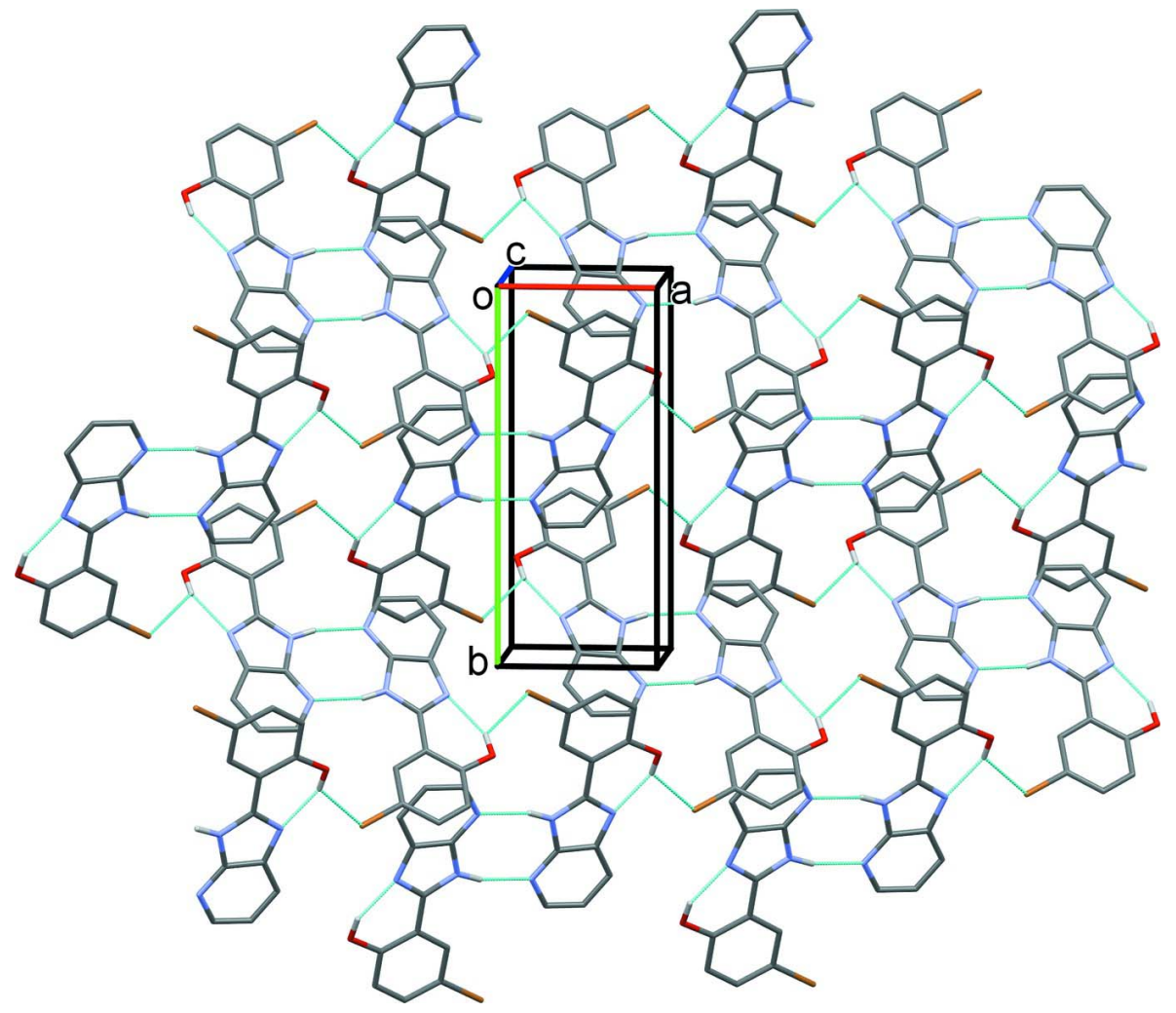

Figure 2

A view along the $c$ axis of the crystal packing of the title compound. The hydrogen bonds are shown as dashed lines (see Table 1), and $\mathrm{H}$ atoms not involved in these interactions have been omitted for clarity. 
Crystal data

$\mathrm{C}_{12} \mathrm{H}_{8} \mathrm{BrN}_{3} \mathrm{O}$

$M_{r}=290.12$

Monoclinic, $P 2_{1} / c$

Hall symbol: -P $2 \mathrm{ybc}$

$a=5.5906(3) \AA$

$b=12.9032(7) \AA$

$c=14.7622(6) \AA$

$\beta=102.836(3)^{\circ}$

$V=1038.28(9) \AA^{3}$

$Z=4$

Data collection

Nonius KappaCCD diffractometer

Radiation source: sealed tube

Graphite monochromator

phi and $\omega$ scans

Absorption correction: multi-scan

(MULABS in PLATON; Spek, 2009)

$T_{\min }=0.457, T_{\max }=0.721$

\section{Refinement}

Refinement on $F^{2}$

Least-squares matrix: full

$R\left[F^{2}>2 \sigma\left(F^{2}\right)\right]=0.043$

$w R\left(F^{2}\right)=0.111$

$S=1.02$

3017 reflections

159 parameters

0 restraints

Primary atom site location: structure-invariant

direct methods
$F(000)=576$

$D_{\mathrm{x}}=1.856 \mathrm{Mg} \mathrm{m}^{-3}$

Mo $K \alpha$ radiation, $\lambda=0.71073 \AA$

Cell parameters from 4475 reflections

$\theta=1.0-30.0^{\circ}$

$\mu=3.94 \mathrm{~mm}^{-1}$

$T=193 \mathrm{~K}$

Plate, orange

$0.25 \times 0.20 \times 0.05 \mathrm{~mm}$

8584 measured reflections

3017 independent reflections

1977 reflections with $I>2 \sigma(I)$

$R_{\text {int }}=0.066$

$\theta_{\text {max }}=30.0^{\circ}, \theta_{\min }=2.1^{\circ}$

$h=-7 \rightarrow 4$

$k=-17 \rightarrow 18$

$l=-20 \rightarrow 19$

Secondary atom site location: difference Fourier map

Hydrogen site location: inferred from

neighbouring sites

$\mathrm{H}$ atoms treated by a mixture of independent and constrained refinement

$w=1 /\left[\sigma^{2}\left(F_{\mathrm{o}}^{2}\right)+(0.045 P)^{2}+0.5334 P\right]$

where $P=\left(F_{\mathrm{o}}^{2}+2 F_{\mathrm{c}}^{2}\right) / 3$

$(\Delta / \sigma)_{\max }=0.002$

$\Delta \rho_{\max }=0.52$ e $\AA^{-3}$

$\Delta \rho_{\min }=-0.84$ e $\AA^{-3}$

\section{Special details}

Geometry. All s.u.'s (except the s.u. in the dihedral angle between two 1.s. planes) are estimated using the full covariance matrix. The cell s.u.'s are taken into account individually in the estimation of s.u.'s in distances, angles and torsion angles; correlations between s.u.'s in cell parameters are only used when they are defined by crystal symmetry. An approximate (isotropic) treatment of cell s.u.'s is used for estimating s.u.'s involving l.s. planes.

Refinement. Refinement of $F^{2}$ against ALL reflections. The weighted $R$-factor $w R$ and goodness of fit $S$ are based on $F^{2}$, conventional $R$-factors $R$ are based on $F$, with $F$ set to zero for negative $F^{2}$. The threshold expression of $F^{2}>2 \sigma\left(F^{2}\right)$ is used only for calculating $R$-factors(gt) etc. and is not relevant to the choice of reflections for refinement. $R$-factors based on $F^{2}$ are statistically about twice as large as those based on $F$, and $R$ - factors based on ALL data will be even larger.

Fractional atomic coordinates and isotropic or equivalent isotropic displacement parameters $\left(\hat{A}^{2}\right)$

\begin{tabular}{lllll}
\hline & $x$ & $y$ & $z$ & $U_{\text {iso }} * / U_{\text {eq }}$ \\
\hline Br1 & $0.83482(7)$ & $0.58489(3)$ & $1.18198(2)$ & $0.03255(13)$ \\
O1 & $0.0419(4)$ & $0.75774(17)$ & $0.87477(15)$ & $0.0274(5)$ \\
H1 & 0.0891 & 0.8104 & 0.8502 & $0.041^{*}$
\end{tabular}




$\begin{array}{lllll}\mathrm{N} 1 & 0.7079(5) & 0.9288(2) & 0.95213(18) & 0.0226(6) \\ \mathrm{N} 2 & 0.3311(5) & 0.91524(19) & 0.85876(18) & 0.0249(6) \\ \mathrm{N} 3 & 0.8399(5) & 1.09033(19) & 0.89394(18) & 0.0242(6) \\ \mathrm{C} 1 & 0.4545(6) & 0.7733(2) & 0.9701(2) & 0.0231(7) \\ \mathrm{C} 2 & 0.6365(6) & 0.7295(2) & 1.0408(2) & 0.0248(7) \\ \mathrm{H} 2 & 0.7917 & 0.7625 & 1.0592 & 0.030^{*} \\ \mathrm{C} 3 & 0.5911(6) & 0.6389(2) & 1.0835(2) & 0.0245(7) \\ \mathrm{C} 4 & 0.3660(6) & 0.5889(2) & 1.0569(2) & 0.0276(7) \\ \mathrm{H} 4 & 0.3362 & 0.5266 & 1.0868 & 0.033^{*} \\ \mathrm{C} 5 & 0.1866(6) & 0.6303(3) & 0.9869(2) & 0.0299(8) \\ \mathrm{H} 5 & 0.0333 & 0.5959 & 0.9684 & 0.036^{*} \\ \mathrm{C} 6 & 0.2276(6) & 0.7220(2) & 0.9429(2) & 0.0247(7) \\ \mathrm{C} 7 & 0.4972(6) & 0.8720(2) & 0.9272(2) & 0.0229(7) \\ \mathrm{C} 8 & 0.6765(6) & 1.0143(2) & 0.8952(2) & 0.0222(6) \\ \mathrm{C} 9 & 0.4404(6) & 1.0061(2) & 0.8373(2) & 0.0233(7) \\ \mathrm{C} 10 & 0.3592(6) & 1.0841(2) & 0.7730(2) & 0.0265(7) \\ \mathrm{H} 10 & 0.1997 & 1.0834 & 0.7336 & 0.032^{*} \\ \mathrm{C} 11 & 0.5249(7) & 1.1633(2) & 0.7697(2) & 0.0287(7) \\ \mathrm{H} 11 & 0.4796 & 1.2182 & 0.7264 & 0.034^{*} \\ \mathrm{C} 12 & 0.7572(6) & 1.1635(3) & 0.8290(2) & 0.0281(7) \\ \mathrm{H} 12 & 0.8647 & 1.2189 & 0.8233 & 0.034^{*} \\ \mathrm{H} 1 \mathrm{~N} & 0.843(8) & 0.912(3) & 0.998(3) & 0.034(10)^{*}\end{array}$

Atomic displacement parameters $\left(\AA^{2}\right)$

\begin{tabular}{lllllll}
\hline & $U^{11}$ & $U^{22}$ & $U^{33}$ & $U^{12}$ & $U^{13}$ & $U^{23}$ \\
\hline Br1 & $0.0314(2)$ & $0.0314(2)$ & $0.0320(2)$ & $-0.00049(16)$ & $0.00116(14)$ & $0.00779(15)$ \\
O1 & $0.0181(12)$ & $0.0281(12)$ & $0.0317(12)$ & $-0.0034(9)$ & $-0.0036(9)$ & $0.0059(10)$ \\
N1 & $0.0197(14)$ & $0.0238(14)$ & $0.0231(13)$ & $-0.0013(11)$ & $0.0025(11)$ & $0.0028(11)$ \\
N2 & $0.0211(14)$ & $0.0251(14)$ & $0.0264(13)$ & $-0.0024(12)$ & $0.0010(11)$ & $-0.0003(11)$ \\
N3 & $0.0236(14)$ & $0.0224(14)$ & $0.0270(14)$ & $-0.0024(11)$ & $0.0063(11)$ & $0.0007(11)$ \\
C1 & $0.0212(17)$ & $0.0236(16)$ & $0.0258(16)$ & $-0.0015(12)$ & $0.0079(13)$ & $-0.0015(13)$ \\
C2 & $0.0195(17)$ & $0.0261(17)$ & $0.0281(16)$ & $-0.0050(13)$ & $0.0038(13)$ & $-0.0016(13)$ \\
C3 & $0.0252(18)$ & $0.0223(16)$ & $0.0253(16)$ & $0.0027(13)$ & $0.0037(13)$ & $0.0003(13)$ \\
C4 & $0.0292(18)$ & $0.0198(15)$ & $0.0343(18)$ & $-0.0048(14)$ & $0.0079(14)$ & $0.0019(14)$ \\
C5 & $0.0261(19)$ & $0.0254(18)$ & $0.0381(19)$ & $-0.0082(14)$ & $0.0070(15)$ & $-0.0064(15)$ \\
C6 & $0.0212(17)$ & $0.0274(17)$ & $0.0257(16)$ & $-0.0006(13)$ & $0.0055(13)$ & $-0.0049(13)$ \\
C7 & $0.0192(16)$ & $0.0259(16)$ & $0.0235(15)$ & $-0.0018(13)$ & $0.0044(13)$ & $-0.0017(13)$ \\
C8 & $0.0212(16)$ & $0.0251(16)$ & $0.0211(15)$ & $0.0012(13)$ & $0.0067(12)$ & $-0.0042(13)$ \\
C9 & $0.0220(17)$ & $0.0248(16)$ & $0.0235(15)$ & $-0.0014(13)$ & $0.0056(13)$ & $-0.0007(13)$ \\
C10 & $0.0232(17)$ & $0.0309(17)$ & $0.0232(15)$ & $0.0006(15)$ & $0.0004(12)$ & $-0.0014(14)$ \\
C11 & $0.035(2)$ & $0.0248(17)$ & $0.0261(17)$ & $0.0003(15)$ & $0.0066(14)$ & $0.0036(14)$ \\
C12 & $0.0287(18)$ & $0.0255(17)$ & $0.0315(18)$ & $-0.0025(14)$ & $0.0100(14)$ & $0.0014(14)$ \\
& & & & & &
\end{tabular}

Geometric parameters $(\AA, \stackrel{\circ}{)})$

\begin{tabular}{llll}
\hline $\mathrm{Br} 1-\mathrm{C} 3$ & $1.891(3)$ & $\mathrm{C} 2-\mathrm{H} 2$ & 0.9500 \\
$\mathrm{O} 1-\mathrm{C} 6$ & $1.356(4)$ & $\mathrm{C} 3-\mathrm{C} 4$ & $1.391(5)$
\end{tabular}




\begin{tabular}{|c|c|c|c|}
\hline $\mathrm{O} 1-\mathrm{H} 1$ & 0.8400 & $\mathrm{C} 4-\mathrm{C} 5$ & $1.378(5)$ \\
\hline $\mathrm{N} 1-\mathrm{C} 7$ & $1.366(4)$ & $\mathrm{C} 4-\mathrm{H} 4$ & 0.9500 \\
\hline $\mathrm{N} 1-\mathrm{C} 8$ & $1.375(4)$ & $\mathrm{C} 5-\mathrm{C} 6$ & $1.393(5)$ \\
\hline $\mathrm{N} 1-\mathrm{H} 1 \mathrm{~N}$ & $0.92(4)$ & $\mathrm{C} 5-\mathrm{H} 5$ & 0.9500 \\
\hline $\mathrm{N} 2-\mathrm{C} 7$ & $1.333(4)$ & $\mathrm{C} 8-\mathrm{C} 9$ & $1.407(4)$ \\
\hline $\mathrm{N} 2-\mathrm{C} 9$ & $1.390(4)$ & $\mathrm{C} 9-\mathrm{C} 10$ & $1.390(4)$ \\
\hline N3-C8 & $1.344(4)$ & $\mathrm{C} 10-\mathrm{C} 11$ & $1.387(5)$ \\
\hline $\mathrm{N} 3-\mathrm{C} 12$ & $1.352(4)$ & $\mathrm{C} 10-\mathrm{H} 10$ & 0.9500 \\
\hline $\mathrm{C} 1-\mathrm{C} 2$ & $1.405(4)$ & $\mathrm{C} 11-\mathrm{C} 12$ & $1.395(5)$ \\
\hline $\mathrm{C} 1-\mathrm{C} 6$ & $1.408(4)$ & $\mathrm{C} 11-\mathrm{H} 11$ & 0.9500 \\
\hline $\mathrm{C} 1-\mathrm{C} 7$ & $1.465(4)$ & $\mathrm{C} 12-\mathrm{H} 12$ & 0.9500 \\
\hline $\mathrm{C} 2-\mathrm{C} 3$ & $1.378(4)$ & & \\
\hline $\mathrm{C} 6-\mathrm{O} 1-\mathrm{H} 1$ & 109.5 & $\mathrm{O} 1-\mathrm{C} 6-\mathrm{C} 5$ & $117.2(3)$ \\
\hline $\mathrm{C} 7-\mathrm{N} 1-\mathrm{C} 8$ & $106.2(3)$ & $\mathrm{O} 1-\mathrm{C} 6-\mathrm{C} 1$ & $123.0(3)$ \\
\hline $\mathrm{C} 7-\mathrm{N} 1-\mathrm{H} 1 \mathrm{~N}$ & $126(2)$ & $\mathrm{C} 5-\mathrm{C} 6-\mathrm{C} 1$ & $119.9(3)$ \\
\hline $\mathrm{C} 8-\mathrm{N} 1-\mathrm{H} 1 \mathrm{~N}$ & $127(2)$ & $\mathrm{N} 2-\mathrm{C} 7-\mathrm{N} 1$ & $113.2(3)$ \\
\hline $\mathrm{C} 7-\mathrm{N} 2-\mathrm{C} 9$ & $104.9(3)$ & $\mathrm{N} 2-\mathrm{C} 7-\mathrm{C} 1$ & $122.6(3)$ \\
\hline $\mathrm{C} 8-\mathrm{N} 3-\mathrm{C} 12$ & $113.1(3)$ & $\mathrm{N} 1-\mathrm{C} 7-\mathrm{C} 1$ & $124.3(3)$ \\
\hline $\mathrm{C} 2-\mathrm{C} 1-\mathrm{C} 6$ & $118.7(3)$ & $\mathrm{N} 3-\mathrm{C} 8-\mathrm{N} 1$ & $126.8(3)$ \\
\hline $\mathrm{C} 2-\mathrm{C} 1-\mathrm{C} 7$ & $120.7(3)$ & $\mathrm{N} 3-\mathrm{C} 8-\mathrm{C} 9$ & $126.6(3)$ \\
\hline $\mathrm{C} 6-\mathrm{C} 1-\mathrm{C} 7$ & $120.6(3)$ & $\mathrm{N} 1-\mathrm{C} 8-\mathrm{C} 9$ & $106.6(3)$ \\
\hline $\mathrm{C} 3-\mathrm{C} 2-\mathrm{C} 1$ & $120.3(3)$ & $\mathrm{C} 10-\mathrm{C} 9-\mathrm{N} 2$ & $132.3(3)$ \\
\hline $\mathrm{C} 3-\mathrm{C} 2-\mathrm{H} 2$ & 119.8 & $\mathrm{C} 10-\mathrm{C} 9-\mathrm{C} 8$ & $118.7(3)$ \\
\hline $\mathrm{C} 1-\mathrm{C} 2-\mathrm{H} 2$ & 119.8 & $\mathrm{~N} 2-\mathrm{C} 9-\mathrm{C} 8$ & $109.0(3)$ \\
\hline $\mathrm{C} 2-\mathrm{C} 3-\mathrm{C} 4$ & $120.8(3)$ & $\mathrm{C} 11-\mathrm{C} 10-\mathrm{C} 9$ & $116.0(3)$ \\
\hline $\mathrm{C} 2-\mathrm{C} 3-\mathrm{Br} 1$ & $119.4(2)$ & $\mathrm{C} 11-\mathrm{C} 10-\mathrm{H} 10$ & 122.0 \\
\hline $\mathrm{C} 4-\mathrm{C} 3-\mathrm{Br} 1$ & $119.9(2)$ & $\mathrm{C} 9-\mathrm{C} 10-\mathrm{H} 10$ & 122.0 \\
\hline $\mathrm{C} 5-\mathrm{C} 4-\mathrm{C} 3$ & $119.6(3)$ & $\mathrm{C} 10-\mathrm{C} 11-\mathrm{C} 12$ & $121.0(3)$ \\
\hline $\mathrm{C} 5-\mathrm{C} 4-\mathrm{H} 4$ & 120.2 & $\mathrm{C} 10-\mathrm{C} 11-\mathrm{H} 11$ & 119.5 \\
\hline $\mathrm{C} 3-\mathrm{C} 4-\mathrm{H} 4$ & 120.2 & $\mathrm{C} 12-\mathrm{C} 11-\mathrm{H} 11$ & 119.5 \\
\hline $\mathrm{C} 4-\mathrm{C} 5-\mathrm{C} 6$ & $120.7(3)$ & $\mathrm{N} 3-\mathrm{C} 12-\mathrm{C} 11$ & $124.6(3)$ \\
\hline $\mathrm{C} 4-\mathrm{C} 5-\mathrm{H} 5$ & 119.6 & $\mathrm{~N} 3-\mathrm{C} 12-\mathrm{H} 12$ & 117.7 \\
\hline $\mathrm{C} 6-\mathrm{C} 5-\mathrm{H} 5$ & 119.6 & $\mathrm{C} 11-\mathrm{C} 12-\mathrm{H} 12$ & 117.7 \\
\hline $\mathrm{C} 6-\mathrm{C} 1-\mathrm{C} 2-\mathrm{C} 3$ & $-1.1(5)$ & $\mathrm{C} 6-\mathrm{C} 1-\mathrm{C} 7-\mathrm{N} 2$ & $-3.0(5)$ \\
\hline $\mathrm{C} 7-\mathrm{C} 1-\mathrm{C} 2-\mathrm{C} 3$ & $177.2(3)$ & $\mathrm{C} 2-\mathrm{C} 1-\mathrm{C} 7-\mathrm{N} 1$ & $-1.0(5)$ \\
\hline $\mathrm{C} 1-\mathrm{C} 2-\mathrm{C} 3-\mathrm{C} 4$ & $0.6(5)$ & $\mathrm{C} 6-\mathrm{C} 1-\mathrm{C} 7-\mathrm{N} 1$ & $177.3(3)$ \\
\hline $\mathrm{C} 1-\mathrm{C} 2-\mathrm{C} 3-\mathrm{Br} 1$ & $-177.4(2)$ & $\mathrm{C} 12-\mathrm{N} 3-\mathrm{C} 8-\mathrm{N} 1$ & $179.0(3)$ \\
\hline $\mathrm{C} 2-\mathrm{C} 3-\mathrm{C} 4-\mathrm{C} 5$ & $0.1(5)$ & $\mathrm{C} 12-\mathrm{N} 3-\mathrm{C} 8-\mathrm{C} 9$ & $0.0(5)$ \\
\hline $\mathrm{Br} 1-\mathrm{C} 3-\mathrm{C} 4-\mathrm{C} 5$ & $178.1(3)$ & $\mathrm{C} 7-\mathrm{N} 1-\mathrm{C} 8-\mathrm{N} 3$ & $-178.5(3)$ \\
\hline $\mathrm{C} 3-\mathrm{C} 4-\mathrm{C} 5-\mathrm{C} 6$ & $-0.4(5)$ & $\mathrm{C} 7-\mathrm{N} 1-\mathrm{C} 8-\mathrm{C} 9$ & $0.7(3)$ \\
\hline $\mathrm{C} 4-\mathrm{C} 5-\mathrm{C} 6-\mathrm{O} 1$ & $-179.9(3)$ & $\mathrm{C} 7-\mathrm{N} 2-\mathrm{C} 9-\mathrm{C} 10$ & $-179.5(3)$ \\
\hline $\mathrm{C} 4-\mathrm{C} 5-\mathrm{C} 6-\mathrm{C} 1$ & $-0.1(5)$ & $\mathrm{C} 7-\mathrm{N} 2-\mathrm{C} 9-\mathrm{C} 8$ & $0.3(4)$ \\
\hline $\mathrm{C} 2-\mathrm{C} 1-\mathrm{C} 6-\mathrm{O} 1$ & $-179.4(3)$ & $\mathrm{N} 3-\mathrm{C} 8-\mathrm{C} 9-\mathrm{C} 10$ & $-1.6(5)$ \\
\hline $\mathrm{C} 7-\mathrm{C} 1-\mathrm{C} 6-\mathrm{O} 1$ & $2.3(5)$ & $\mathrm{N} 1-\mathrm{C} 8-\mathrm{C} 9-\mathrm{C} 10$ & $179.2(3)$ \\
\hline $\mathrm{C} 2-\mathrm{C} 1-\mathrm{C} 6-\mathrm{C} 5$ & $0.8(5)$ & $\mathrm{N} 3-\mathrm{C} 8-\mathrm{C} 9-\mathrm{N} 2$ & $178.6(3)$ \\
\hline $\mathrm{C} 7-\mathrm{C} 1-\mathrm{C} 6-\mathrm{C} 5$ & $-177.5(3)$ & $\mathrm{N} 1-\mathrm{C} 8-\mathrm{C} 9-\mathrm{N} 2$ & $-0.6(4)$ \\
\hline
\end{tabular}




$\begin{array}{llll}\mathrm{C} 9-\mathrm{N} 2-\mathrm{C} 7-\mathrm{N} 1 & 0.2(4) & \mathrm{N} 2-\mathrm{C} 9-\mathrm{C} 10-\mathrm{C} 11 & -178.4(3) \\ \mathrm{C} 9-\mathrm{N} 2-\mathrm{C} 7-\mathrm{C} 1 & -179.6(3) & \mathrm{C} 8-\mathrm{C} 9-\mathrm{C} 10-\mathrm{C} 11 & 1.9(4) \\ \mathrm{C} 8-\mathrm{N} 1-\mathrm{C} 7-\mathrm{N} 2 & -0.6(4) & \mathrm{C} 9-\mathrm{C} 10-\mathrm{C} 11-\mathrm{C} 12 & -0.8(5) \\ \mathrm{C} 8-\mathrm{N} 1-\mathrm{C} 7-\mathrm{C} 1 & 179.2(3) & \mathrm{C} 8-\mathrm{N} 3-\mathrm{C} 12-\mathrm{C} 11 & 1.3(5) \\ \mathrm{C} 2-\mathrm{C} 1-\mathrm{C} 7-\mathrm{N} 2 & 178.7(3) & \mathrm{C} 10-\mathrm{C} 11-\mathrm{C} 12-\mathrm{N} 3 & -0.9(5)\end{array}$

Hydrogen-bond geometry $\left(\AA,{ }^{\circ}\right)$

\begin{tabular}{lllll}
\hline$D-\mathrm{H} \cdots A$ & $D-\mathrm{H}$ & $\mathrm{H} \cdots A$ & $D \cdots A$ & $D-\mathrm{H} \cdots A$ \\
\hline $\mathrm{O} 1-\mathrm{H} 1 \cdots \mathrm{N} 2$ & 0.84 & 1.90 & $2.640(3)$ & 147 \\
$\mathrm{O} 1-\mathrm{H} 1 \cdots \mathrm{Br} 1^{\mathrm{i}}$ & 0.84 & 2.91 & $3.478(2)$ & 127 \\
$\mathrm{~N} 1-\mathrm{H} 1 N \cdots \mathrm{N} 3^{\mathrm{ii}}$ & $0.92(4)$ & $2.11(4)$ & $3.010(4)$ & $168(3)$ \\
\hline
\end{tabular}

Symmetry codes: (i) $x-1,-y+3 / 2, z-1 / 2$; (ii) $-x+2,-y+2,-z+2$. 